\title{
Mitochondrial Oxidative Stress Impairs Energy Metabolism and Reduces Stress Resistance and Longevity of C. elegans
}

\author{
Benjamin Dilberger, ${ }^{1}$ Stefan Baumanns, ${ }^{2}$ Fabian Schmitt, ${ }^{1}$ Tommy Schmiedl, ${ }^{1}$ \\ Martin Hardt, ${ }^{3}$ Uwe Wenzel, ${ }^{2}$ and Gunter P. Eckert ${ }^{1}{ }^{1}$ \\ ${ }^{1}$ Institute of Nutritional Sciences, Laboratory for Nutrition in Prevention and Therapy, Biomedical Research Center Seltersberg (BFS), \\ Justus Liebig University Giessen, Schubertstrasse 81, 35392 Giessen, Germany \\ ${ }^{2}$ Molecular Nutrition Research, Interdisciplinary Research Center, Justus Liebig University Giessen, Heinrich-Buff-Ring 26-32, \\ 35392 Giessen, Germany \\ ${ }^{3}$ Imaging Unit, Biomedical Research Center Seltersberg (BFS), Justus Liebig University Giessen, Schubertstrasse 81, \\ 35392 Giessen, Germany
}

Correspondence should be addressed to Gunter P. Eckert; eckert@uni-giessen.de

Received 6 August 2019; Revised 8 October 2019; Accepted 22 October 2019; Published 15 November 2019

Academic Editor: Ravirajsinh Jadeja

Copyright (c) 2019 Benjamin Dilberger et al. This is an open access article distributed under the Creative Commons Attribution License, which permits unrestricted use, distribution, and reproduction in any medium, provided the original work is properly cited.

\begin{abstract}
Introduction. Mitochondria supply cellular energy and are key regulators of intrinsic cell death and consequently affect longevity. The nematode Caenorhabditis elegans is frequently used for lifespan assays. Using paraquat (PQ) as a generator of reactive oxygen species, we here describe its effects on the acceleration of aging and the associated dysfunctions at the level of mitochondria. Methods. Nematodes were incubated with various concentrations of paraquat in a heat-stress resistance assay ( $\left.37^{\circ} \mathrm{C}\right)$ using nucleic staining. The most effective concentration was validated under physiological conditions, and chemotaxis was assayed. Mitochondrial membrane potential $(\Delta \Psi \mathrm{m})$ was measured using rhodamine 123 , and activity of respiratory chain complexes determined using a Clark-type electrode in isolated mitochondria. Energetic metabolites in the form of pyruvate, lactate, and ATP were determined using commercial kits. Mitochondrial integrity and structure was investigated using transmission electron microscopy. Live imaging after staining with fluorescent dyes was used to measure mitochondrial and cytosolic ROS. Expression of longevity- and mitogenesis-related genes were evaluated using qRT-PCR. Results. PQ (5 mM) significantly increased ROS formation in nematodes and reduced the chemotaxis, the physiological lifespan, and the survival in assays for heat-stress resistance. The number of fragmented mitochondria significantly increased. The $\Delta \Psi \mathrm{m}$, the activities of complexes I-IV of the mitochondrial respiratory chain, and the levels of pyruvate and lactate were significantly reduced, whereas ATP production was not affected. Transcript levels of genetic marker genes, atfs-1, atp-2, skn-1, and sir-2.1, were significantly upregulated after PQ incubation, which implicates a close connection between mitochondrial dysfunction and oxidative stress response. Expression levels of aak-2 and daf-16 were unchanged. Conclusion. Using paraquat as a stressor, we here describe the association of oxidative stress, restricted energy metabolism, and reduced stress resistance and longevity in the nematode Caenorhabditis elegans making it a readily accessible in vivo model for mitochondrial dysfunction.
\end{abstract}

\section{Introduction}

Mitochondria supply cellular energy and are key regulators of intrinsic cell death and consequently affect longevity $[1,2]$. A link between aging and mitochondrial dysfunction has been well established [2-7]. The "free radical theory" of aging, first proposed by Harman et al., explains aging as a result of the accumulation of cellular damage caused by reactive oxygen species (ROS) $[2,8,9]$. Since mitochondria are the primary source of ROS, Harman himself extended his theory to the "mitochondrial theory of aging" [3, 10]. An imbalance between ROS and cellular stress defence mechanisms accordingly causes a vicious cycle of further mitochondrial dysfunction leading to more ROS, which in turn 
promotes more damage, an energetic imbalance, and finally triggers cell death and thereby aging. The importance of an equilibrium between ROS and defence mechanism is evidenced by the fact that low concentrations of ROS lead to hormesis with a higher state of stress resistance [11-14].

Investigations on isolated mitochondria in aging nematodes are scarce. Several organisms, ranging from yeast to mice $[13,15-18]$, have been used to study the effect of alterations within the mitochondrial electron chain (ETC) and longevity [5]. However, C. elegans offers distinct advantages compared to other model organisms. Especially its ability as a hermaphrodite to produce identical offspring and its short lifespan make it a powerful tool that has been widely used to investigate longevity-related questions $[19,20]$. Since molecular and functional processes associated with mitochondria are highly conserved in species over long evolutionary distances, C. elegans represents an outstanding model for aging mechanisms with mitochondria [20]. Specific transcription factors, including $s k n-1$ (Nrf-2 ortholog), aak-2 (AMPK ortholog), atfs-1, and sir-2.1 (Sirt1 ortholog), involved in crucial metabolic pathways [21-24], have been identified, connecting alterations in longevity to mitochondrial dysfunction and mitochondrial biogenesis [25, 26].

Compared to in situ studies, investigating isolated mitochondria in the context of aging, however, appears to be crucial since it offers clear advantages [27] in an environment free from interfering organelles or reactions [28].

In the present study, paraquat $\left(1,1^{\prime}\right.$-dimethyl-4' 4 -bipyridinium dichloride; PQ) was used as a well-known stressor of the mitochondrial respiration chain in order to assess the effects of mitochondrial dysfunction on stress resistance and aging. Life span and health span of nematodes, generation of mitochondrial and cytosolic ROS, energy metabolites (ATP, lactate, and pyruvate), and expression of key genes were investigated in whole animals. Mitochondrial integrity and structure as well as activities of ETC complexes and membrane potential $(\Delta \Psi \mathrm{m})$ were evaluated in isolated mitochondria putting the close relation between mitochondrial dysfunction and longevity into a broader context.

\section{Material and Methods}

2.1. Chemicals. Chemicals used were of the highest available purity and standard from Sigma-Aldrich (St. Louis, MO, USA) or Merck (Darmstadt, Germany).

2.2. Nematode and Bacterial Strain. C. elegans wild-type strain N2 was obtained from the Caenorhabditis Genetics Center (University of Minnesota, MN, US). Nematodes were maintained on nematode growth medium (NGM) agar plates seeded with E. coli OP50 at $20^{\circ} \mathrm{C}$ according to standard protocols [29]. For all experiments, synchronous populations were generated through a standard bleaching protocol [30].

2.3. Cultivation and Treatment. Synchronous larvae were washed twice in M9 buffer, counted, and adjusted to 10 larvae per $10 \mu \mathrm{L}$. Depending on the experiment and on the number needed, nematodes were either raised in 96-well plates (Greiner Bio-One, Frickenhausen, Germany), cell culture flasks (Sarstedt, Nümbrecht, Germany), or OP50 spread NGM plates. For 96-well plates and flasks, OP50-NGM was added as a standardized food source with a volume 4.4-fold of the larvae containing M9 solution. L1 larvae were maintained under shaking at $20^{\circ} \mathrm{C}$ reaching adulthood within 3 days.

Unless otherwise stated, paraquat was dissolved in M9 and added after reaching young adulthood, $48 \mathrm{~h}$ prior to the experiment. M9 was used as control.

2.4. Lifespan Assay. To determine the nematode's lifespan at $20^{\circ} \mathrm{C}$, a modified protocol from Amrit et al. was applied [31] and synchronized larvae, obtained from egg preparation as stated above, were raised on NGM agar plates spread with standard OP50 E. coli culture. After completing the L4 larval stage, 60 healthy animals per group were transferred to fresh NGM E. coli containing plates with a sterilized platinum wire. Effectors were incorporated into the OP50 culture with the concentration as needed. Nematodes were transferred to new plates every two days to distinguish between offspring until egg-laying stopped. In line with the separation from eggs and larvae, nematodes were checked for vital signs using a hot platinum wire held next to the animals' heads. Worms showing no reaction to the heat stimulus were considered dead. The lifespan curves were statistically compared using the log-rank test.

2.5. Heat-Shock Survival Assay. Approximately 10 nematodes were raised per well in a 96-well microplate as mentioned above. After $48 \mathrm{~h}$ of incubation with effectors, time till death was determined using a microplate thermotolerance assay [32]. In brief, nematodes were washed off the wells with M9-buffer into $15 \mathrm{~mL}$ tubes followed by three additional washing steps. Each well of a black 384-well low-volume microtiter plate (Greiner Bio-One, Frickenhausen, Germany) was prefilled with $6.5 \mu \mathrm{L}$ M9 buffer/Tween ${ }^{\circledR} 20(1 \% v / v)$. Subsequently, one nematode was immersed into $1 \mu \mathrm{L}$ M9 buffer under a stereomicroscope (Breukhoven Microscope Systems, Netherlands). A volume of 7.5 $\mu \mathrm{L}$ SYTOX ${ }^{\mathrm{TM}}$ Green (final concentration $1 \mu \mathrm{M}$; Life Technologies, Karlsruhe, Germany), which penetrates only into cells with compromised plasma membrane and gets fluorescent after binding to DNA, was added for fluorescent detection. To prevent water evaporation, the plates were sealed with a Rotilabo sealing film (Greiner Bio-One, Frickenhausen, Germany). Heat shock $\left(37^{\circ} \mathrm{C}\right)$ was applied and fluorescence measured with a ClarioStar Plate Reader (BMG, Ortenberg, Germany) every $30 \mathrm{~min}$ over the course of $17 \mathrm{~h}$. The excitation wavelength was set at $485 \mathrm{~nm}$, and the emission detected at $538 \mathrm{~nm}$.

2.6. Chemotaxis Assay. Chemotaxis was assessed using a previously published method [33]. Briefly, agar plates were divided into four quadrants. Sodium acid $(0.5 \mathrm{M})$ was mixed in the same parts with ethanol (95\%) as control, or diacetyl $(0.5 \%)$ as attractant. Either $2 \mu \mathrm{L}$ of control or attractant solution was added to the center of two opposite quadrants with the same distance to the middle of the plate. Nematodes were washed and separated from larvae as stated above, and a number of approximately 150 animals placed in the plates' 
center. After $1 \mathrm{~h}$, each quadrant was counted, and a chemotaxis index calculated ((number of attractant - number of control)/number total).

2.7. Mitochondrial and Cytosolic ROS Measurement. To determine mitochondrial ROS levels, young adult nematodes were incubated for $48 \mathrm{~h}$ with $0.5 \mu \mathrm{M}$ MitoTracker ${ }^{\circledR}$ Red $\mathrm{CM}-\mathrm{H}_{2} \mathrm{XRos}$ (Fisher Scientific, Schwerte, Germany). MitoTracker ${ }^{\circledR}$ Red accumulates to a high extend at the inner mitochondrial membrane showing an increased fluorescence upon elevated ROS mainly associated with mitochondria. To detect cytosolic ROS, nematodes were incubated for $4 \mathrm{~h}$ with $25 \mu \mathrm{M}$ fluorescent probe $2^{\prime}, 7^{\prime}$-dichlorofluorescein diacetate (CM- $\mathrm{H}_{2} \mathrm{DCFDA}$ ) (Fisher Scientific, Schwerte, Germany). The probe passively diffuses into cells where it is oxidized by cytosolic reactive oxygen species into its fluorescent form. Paraquat $(5 \mathrm{mM})$ was added $4 \mathrm{~h}$ prior to the experiment for both parameters. For epifluorescence microscopy (EVOS FL digital fluorescence microscope, AMG, Bothell, USA), worms were washed with M9-buffer/Tween ${ }^{\circledR} 20(1 \% v / v)$ solution and anesthetized by addition of $2 \mathrm{mM}$ levamisole. Nematodes were transferred onto a labelled glass slide and covered with a cover slip. The dye was visualized using the EVOS LED Light Cube RFP, with an excitation at $531 \pm 40 \mathrm{~nm}$ and an emission at $593 \pm 40 \mathrm{~nm}$ for MitoTracker ${ }^{\circledR}$ Red and an excitation at $470 \pm 22 \mathrm{~nm}$ and an emission at $525 \pm 50 \mathrm{~nm}$ for $\mathrm{CM}-\mathrm{H}_{2} \mathrm{DCFDA}$. Images were taken at a tenfold magnification. For each group, at least 20 nematodes were photographed. The quantification of fluorescence intensity was done using ImageJ (National Institute of Health (NIH)).

2.8. Isolation of Mitochondria. To isolate functional mitochondria, nematode populations ranging from 5,000 to 10,000 per group were needed. Nematodes were raised in liquid OP50-NGM culture medium and incubated with effectors as stated above under standardized conditions.

Two-day-old gravid adults were separated from larvae and washed to remove residual bacteria using a self-made separation device with a nylon mesh (Dr. Fill ${ }^{\circledR}$, Giessen, Germany) before being transferred to ice-cold isolation buffer (300 mM sucrose, 5 mM TES, $200 \mu$ M EGTA, pH 7.2) [34].

To obtain a mitochondria-enriched fraction, a Balch Homogenizer (Isobiotec, Heidelberg, Germany) was used [35]. Nematodes were gently passed through the homogenizer chamber with $1 \mathrm{~mL}$ glass syringes (SGE Syringe, Trajan, Australia) fitted with a metal luer lock for 5 times. To fracture nematode cuticle, a $12 \mu \mathrm{m}$ ball clearance was applied. The homogenate was centrifuged at $800 \mathrm{~g}$ for 5 minutes at $4^{\circ} \mathrm{C}$ (Heraeus Fresco 21, Thermo Scientific, Langenselbold, Germany) to sediment debris and larger worm fragments. The mitochondria-containing supernatant was collected and centrifuged at $9,000 \mathrm{~g}$ for 10 minutes at $4^{\circ} \mathrm{C}$. The crude mitochondria-containing pellet was resuspended in $70 \mu \mathrm{L}$ swelling buffer (SWB) $(0.2 \mathrm{M}$ sucrose, $10 \mathrm{mM}$ MOPS-Tris, $5 \mathrm{mM}$ succinat, $1 \mathrm{mM} \mathrm{H}_{3} \mathrm{PO}_{4}, 10 \mu \mathrm{M}$ EGTA, $2 \mu \mathrm{M}$ rotenone) for measurement of membrane potential $(\Delta \Psi \mathrm{m})$ [34] or $200 \mu \mathrm{L}$ of mitochondrial respiration medium MirO5 (0.5 mM EGTA, $3 \mathrm{mM} \mathrm{MgCl}_{2}, 60 \mathrm{mM}$ K-lactobionate,

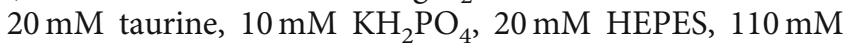

sucrose, $1 \mathrm{~g} / \mathrm{L}$ BSA, pH 7.1; developed by Oroboros) for high-resolution respiratory experiments [36]. For $\Delta \Psi \mathrm{m}$ and respiration measurements, fresh mitochondria were immediately used after preparation. Aliquots were shock frozen in liquid nitrogen for determination of citrate synthase activity and protein content.

2.9. Transmission Electron Microscopy. For fixation, $200 \mu \mathrm{L}$ of fixative (glutaraldehyde (5\%) in $0.1 \mathrm{M}$ cacodylate buffer) was added in $200 \mu \mathrm{L}$ immersed mitochondria, incubated for 30 minutes at room temperature, centrifuged at 9,000 $\mathrm{g}$ for 10 minutes, and replaced with fresh fixative (glutaraldehyde (2.5\%) in $0.1 \mathrm{M}$ cacodylate buffer). Samples were stored under constant movement overnight at $4^{\circ} \mathrm{C}$. Probes were postfixed in $1 \% \mathrm{OsO}_{4}$ in $0.1 \mathrm{M}$ cacodylate buffer for 45 minutes at room temperature. Before staining with $1 \%$ uranyl acetate overnight at $4^{\circ} \mathrm{C}$, samples were embedded in low melting temperature gelatine. The gelatine blocks were dehydrated in an ethanol series (10-20 minutes each in 30\%, 50\%, $70 \%, 80 \%, 90 \%, 96 \%, 99 \%$, and $99 \%$ over molecular sieve) on ice followed by propylene oxide before embedding in Epon and hardened at $60^{\circ} \mathrm{C}$ for 24 hours. Probes were cut into $80 \mathrm{~nm}$ slices, transferred on copper mesh grids and stained with aqueous uranyl acetate followed by lead citrate. Grids were examined with a Leo 912 AB Omega Electron Microscope (Carl Zeiss, Oberkochen, Germany).

To exclude eventual bias, ten pictures for every investigated parameter were taken by a third party and randomized before evaluation. For determination of integrity, mitochondria were divided into four categories ("intact," outer membrane appears intact and crista structure is visible (a); "mildly fractured," slight fractures of outer membrane are visible but mitochondria appear overall in a good state (b); "heavily fractured," outer membrane and crista structure appears damaged (c); "fragmented," mitochondria are torn in parts or only fragments of former mitochondria are visible (d)). Categorization was conducted by two independent investigators.

2.10. Mitochondrial Membrane Potential ( $\Delta \Psi m)$. To determine the mitochondrial membrane potential, a modified protocol of Schmitt et al. was applied [34]. The fluorescent dye rhodamine 123 (Rh123) was used to assess the $\Delta \Psi \mathrm{m}$ of $25 \mu \mathrm{L}$ swelling buffer-resuspended isolated mitochondria in a black 96 well-plate with a ClarioStar Plate Reader (BMG, Ortenberg, Germany). To ensure mitochondrial integrity, the membrane potential was measured for 30 minutes, and after reaching equilibrium, $500 \mathrm{nM}$ FCCP was added to evaluate the $\Delta \Psi \mathrm{m}$-dependent effect on the quenching of Rh123. Results were normalized to protein content.

2.11. High-Resolution Respirometry. Respiration experiments were conducted at $20^{\circ} \mathrm{C}$ using a Clark-type electrode (O2k Oxygraph, Oroboros Instruments, Austria). For each measurement, an aliquot of $80 \mu \mathrm{L}$ in MirO5-resuspended mitochondria, as described preciously, was inserted into $2 \mathrm{~mL}$ of air-saturated MirO5-containing electrode chamber. For analysis, the provided DatLab software (Version 7.0.0.2) was used. To determine mitochondrial function, a 
complex protocol (developed by Prof. Erich Gnaiger, Oroboros, Innsbruck, Austria) was applied, as previously stated [37].

2.12. Citrate-Synthase Activity. Frozen and at $-80^{\circ} \mathrm{C}$, stored samples were slowly thawed, and a reaction mix containing

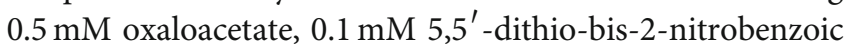
acid (DTNB), $0.31 \mathrm{mM}$ acetyl coenzyme A, $50 \mu \mathrm{M}$ EDTA, $5 \mathrm{mM}$ triethanolamine hydrochloride, and $0.1 \mathrm{M}$ Tris- $\mathrm{HCl}$ was prepared and pre-heated for 5 minutes at $30^{\circ} \mathrm{C}$. To determine citrate synthase (CS) activity, $10 \mu \mathrm{L}$ of mitochondrial suspension was added, and the resulting complex of DNTB with CoA-SH was measured photospectrometrically at $412 \mathrm{~nm}[38,39]$. Measurements were performed in triplicate.

2.13. Nematode Homogenization. To assess energetic metabolites such as ATP, lactate, and pyruvate, a nematode homogenate was generated. In brief, 4,000 synchronized nematodes were harvested, thoroughly washed, shock frozen, and boiled for 15 minutes prior to sonication to denaturate degrading proteins. After centrifugation at $15,000 \mathrm{~g}$ for 10 minutes, supernatants were collected. ATP content was assessed immediately and aliquots stored at $-80^{\circ} \mathrm{C}$ for determination of lactate, pyruvate, and protein content.

2.14. ATP Measurement. Intracellular ATP levels were determined using the ATPlite luminescence assay system (Perkin Elmer, Waltham, MA, USA). Luminescence was measured in triplicate following the manufacturer's guidelines with a ClarioStar Plate Reader (BMG, Ortenberg, Germany). Aliquots were stored at $-80^{\circ} \mathrm{C}$ for determination of protein content.

2.15. Colorimetric Assessment of Lactate and Pyruvate Content. Frozen homogenate samples were slowly thawed until reaching room temperature. Concentrations of lactate and pyruvate were detected by changes in the NADH content using two colorimetric assay kits from Sigma-Aldrich following the manufacturer's guidelines (Sigma-Aldrich, St. Louis, MO, USA) using a ClarioStar Plate Reader (BMG, Ortenberg, Germany).

2.16. Protein Quantification. Protein contents were assessed according to the Pierce ${ }^{\mathrm{TM}}$ BCA Protein Assay Kit (Thermo Fisher Scientific, Waltham, MA, USA). Bovine serum albumin was used as a standard.

2.17. Quantitative Real-Time PCR. Total RNA was isolated using the RNeasy Mini Kit (Qiagen, Hilden, Germany) according to the manufacturer's guidelines after fracturing the nematodes' cuticle using a Balch Homogenizer with $10 \mu \mathrm{M}$ clearance. The concentration of RNA was quantified by measuring the absorbance at 260 and $280 \mathrm{~nm}$ using a NanoDrop $^{\mathrm{TM}}$ 2000c spectrophotometer (Thermo Fisher Scientific, Waltham, MA, USA). RNA purity was assessed with the ratio of absorbance at $260 / 280 \mathrm{~nm}$ and $260 / 230 \mathrm{~nm}$, respectively. Subsequently, samples were treated with a TURBO DNA-free Kit ${ }^{\text {TM }}$ (Thermo Fisher Scientific, Waltham, MA, USA) to remove residual genomic DNA. According to the manufacturer's guidelines, complementary DNA was
TABLE 1: Oligonucleotide primer sequences and product sizes for quantitative real-time PCR. Concentration was $0.1 \mu \mathrm{M}$ for all primers.

\begin{tabular}{|c|c|c|}
\hline Primer & Sequence & Product size (bp) \\
\hline Aak-2 & $\begin{array}{l}5^{\prime} \text {-tgcttcaccatatgctctgc- } 3^{\prime} \\
5^{\prime} \text {-gtggatcatctcccagcaat- } 3^{\prime}\end{array}$ & 219 \\
\hline Ama-1 & $\begin{array}{l}5^{\prime} \text {-ccaggaacttcggctcagta- } 3^{\prime} \\
5^{\prime} \text {-tgtatgatggtgaagctggcg- } 3^{\prime}\end{array}$ & 85 \\
\hline Act-2 & $\begin{array}{l}5^{\prime} \text {-cccactcaatccaaaggcta- } 3^{\prime} \\
5^{\prime} \text {-gggactgtgtgggraacacc- } 3^{\prime}\end{array}$ & 168 \\
\hline Atfs-1 & $\begin{array}{c}5^{\prime} \text {-tcggcgatcgatcagctaac- } 3^{\prime} \\
5^{\prime} \text {-agaatcagttcttggattagggga- } 3^{\prime}\end{array}$ & 75 \\
\hline Atp-2 & $\begin{array}{l}5^{\prime} \text {-tccaagtcgctgaggtgttc- } 3^{\prime} \\
5^{\prime} \text {-aggtggtcgagttctcctga- } 3^{\prime}\end{array}$ & 151 \\
\hline Daf-16 & $\begin{array}{l}5^{\prime} \text {-tcctcattcactcccgattc- } 3^{\prime} \\
5^{\prime} \text {-ccggtgtattcatgaacgtg- } 3^{\prime}\end{array}$ & 175 \\
\hline Sir-2.1 & $\begin{array}{l}5^{\prime} \text {-tggctgacgattcgatggat-3' } \\
5^{\prime} \text {-atgagcagaaatcgcgacac- } 3^{\prime}\end{array}$ & 179 \\
\hline$S k n-1$ & $\begin{array}{l}5^{\prime} \text {-acagggtggaaaaagcaagg- } 3^{\prime} \\
5^{\prime} \text {-caggccaaacgccaatgac- } 3^{\prime}\end{array}$ & 246 \\
\hline
\end{tabular}

bp: base pairs; Conc: concentration.

synthesized from $1 \mu \mathrm{g}$ total RNA using an iScript cDNA Synthesis Kit (Bio-Rad, Munich, Germany) and temporarily stored at $-80^{\circ} \mathrm{C}$. qRT-PCR was conducted using a CfX 96 Connect $^{\mathrm{TM}}$ system (Bio-Rad, Munich, Germany). Primers were purchased from BioMers (Ulm, Germany). Oligonucleotide primer sequences, primer concentrations, and product sizes are listed in Table 1. All cDNA samples were performed in triplicate after a 1:10 dilution with RNase-free water (Quiagen, Hilden, Germany). PCR cycling conditions were an initial denaturation at $95^{\circ} \mathrm{C}$ for $3 \mathrm{~min}$, followed by 45 cycles of $95^{\circ} \mathrm{C}$ for $10 \mathrm{~s}, 58^{\circ} \mathrm{C}$ for $45 \mathrm{~s}$ (with the exception of aak-2 at $62^{\circ} \mathrm{C}$ ), and extension at $72^{\circ} \mathrm{C}$ for $29 \mathrm{~s}$. Gene expression levels were analysed by applying the $-\left(2 \Delta \Delta \mathrm{C}_{\mathrm{q}}\right)$ method using Bio-Rad CfX manager software and normalized to the expression levels of amanitin resistant (ama-1) and actin (act-2).

2.18. Statistics. Values are presented as mean \pm standard error of means (SEM). Statistical analyses were performed by applying Student's $t$-test (Prism 8.0 GraphPad Software, San Diego, CA, USA). Statistical significance was defined for $p$ values ${ }^{*} p<0.05,{ }^{* *} p<0.01,{ }^{* * *} p<0.001$, and ${ }^{* * * *} p<0.0001$.

\section{Results}

3.1. Paraquat Reduces the Survival under Heat-Stress and Lifespan at $20^{\circ} \mathrm{C}$. A screening of various paraquat concentrations showed a dose-dependent decline in heat-stress resistance of nematodes (Figure 1(a)). Exposure to $5 \mathrm{mM}$ paraquat, a concentration which caused a significant 


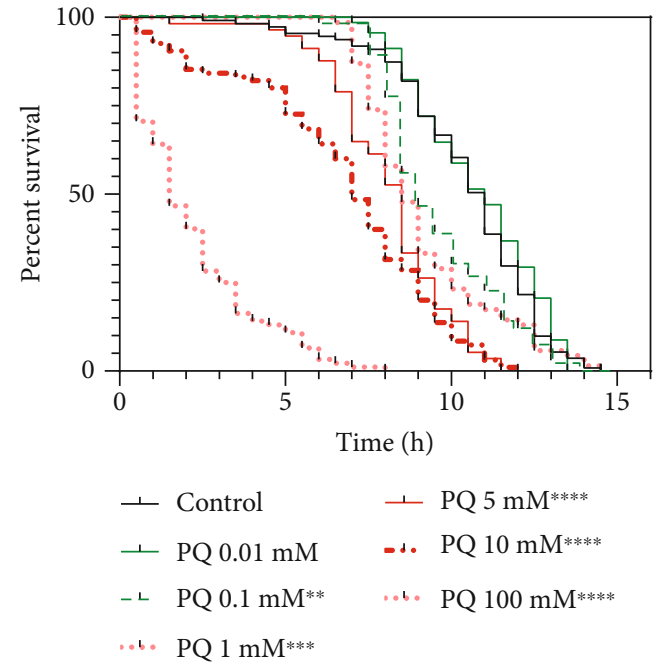

(a)

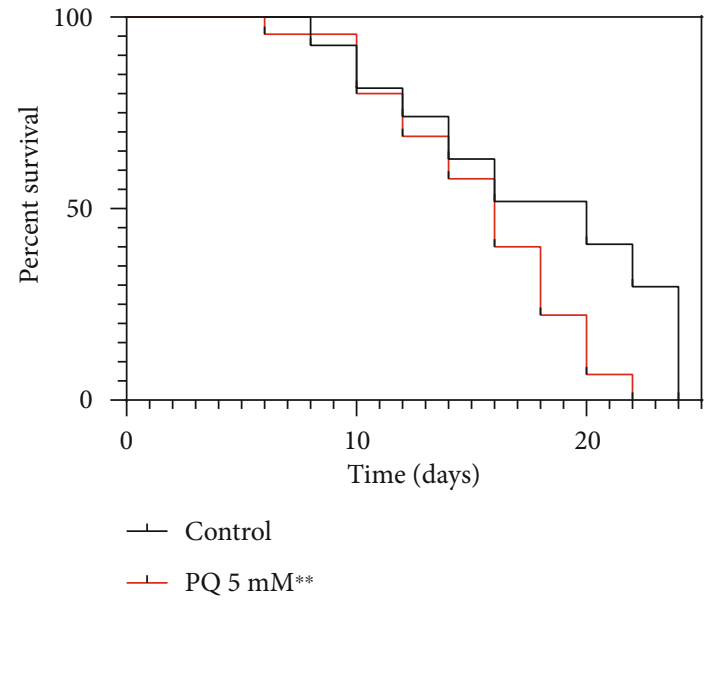

(b)

FIGURE 1: Heat-stress resistance and lifespan of C. elegans are reduced under paraquat exposure. (a) Survival at $37^{\circ} \mathrm{C}$ was assessed due to the penetration of SYTOX ${ }^{\mathrm{TM}}$ Green nucleic acid stain into dead cells. (b) Survival of wild-type C. elegans N2 at $20^{\circ} \mathrm{C}$ was assessed in the absence and presence of $5 \mathrm{mM}$ paraquat. Log-rank (Mantel-Cox) test; ${ }^{* *} p<0.01,{ }^{* * *} p<0.001$, and ${ }^{* * * *} p<0.0001$.

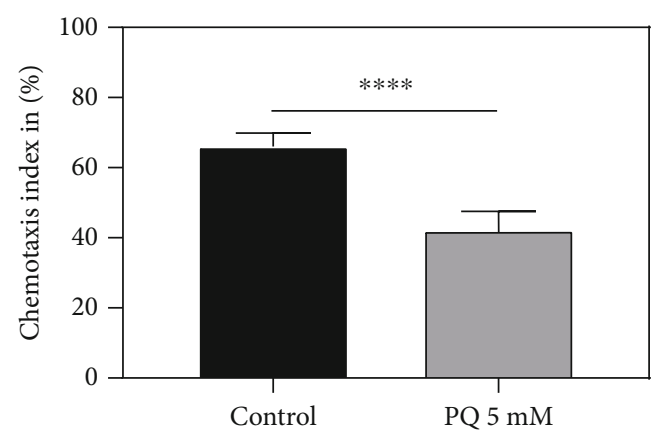

FIgURe 2: Chemotaxis index after treatment with $5 \mathrm{mM}$ PQ for $48 \mathrm{~h}$. $n=5$; mean \pm SEM; Student's $t$-test; ${ }^{* * * *} p<0.0001$.

reduction of stress resistance, also caused a significant reduction in the nematode survival rate at $20^{\circ} \mathrm{C}$ (Figure $1(\mathrm{~b})$ ).

3.2. PQ Reduces Chemotaxis. PQ significantly reduced nematode chemotaxis, resulting in a decreased ability to locate food. Animals insulted with $5 \mathrm{mM} \mathrm{PQ}$ appeared $24 \%$ less likely to locate the attractant diacetyl compared to control $\left({ }^{* * * *} p<0.0001\right)$ (Figure 2).

3.3. Mitochondrial and Cytosolic ROS Measurement. To evaluate the effect of PQ on ROS production in the cytosol and mitochondria, especially, of wild-type nematodes, they were stained with two fluorescent markers. MitoTracker ${ }^{\circledR}$ Red was applied to evaluate mitochondrial site of ROS generation and $\mathrm{CM}-\mathrm{H}_{2} \mathrm{DCFDA}$ for determination of cytosolic ROS (Figure 3).

PQ treatment increases mitochondrial ROS level significantly by $31.5 \%\left({ }^{* * * *} p<0.0001\right)$ and cytosolic ROS level by $19.4 \%\left({ }^{* * * *} p<0.0001\right)$ (Figure 4$)$.
3.4. Effects of $P Q$ on the Integrity of Mitochondria. To elucidate the quality of mitochondria after Balch homogenization as well as the damaging impact of PQ $(5 \mathrm{mM})$ on mitochondrial integrity, we conducted transmission electron mircospy. Figure 5 shows two representative pictures of mitochondria from control nematodes and those treated with $5 \mathrm{mM}$. As visualized, $\mathrm{PQ}$ causes a significant damage of mitochondria, as evidenced by disrupted membranes and loss of crista structure. Exemplary pointers indicate the different degrees of damage as described in Material and Methods. In brief, "intact" (a), mildly "fractured" (b), "heavily fractured" (c), and "fractured" (d) (Figure 5).

Treatment with PQ resulted in a significantly lower number of fully intact and mildly fractured mitochondria compared to the control. For the heavily fractured category, no significant differences could be observed between the two groups, while the number of fragmented mitochondria significantly increased (Figure 6).

3.5. Effect of $P Q$ on Mitochondrial Function. To ensure mitochondrial integrity, its membrane potential $(\Delta \Psi \mathrm{m})$ was measured over the course of 130 minutes after isolation with fluorescent dye rhodamine 123 (Rh123). Addition of $500 \mathrm{nM}$ carbonyl cyanide-4-(trifluoromethoxy)phenylhydrazone (FCCP), at three different time points to aliquots of the same isolation, was used to depolarize the inner mitochondrial membrane and to abolish $\Delta \Psi \mathrm{m}$, after 30,60 , and 120 minutes [34]. Figure 7(a) shows a maximum fluorescence of Rh123 alone and a constant signal after quenching of the fluorescent dye in intact mitochondria beyond the time point of 60 minutes and slowly increasing afterwards. Addition of FCCP depolarizes $\Delta \Psi \mathrm{m}$ resulting in an increased $\mathrm{Rh} 123$ signal after all time points to the same level.

PQ $(5 \mathrm{mM})$ significantly decreased $\Delta \Psi \mathrm{m}$ by $49 \%$ compared to the control (Figure $7(\mathrm{~b})$ ). PQ impaired the 


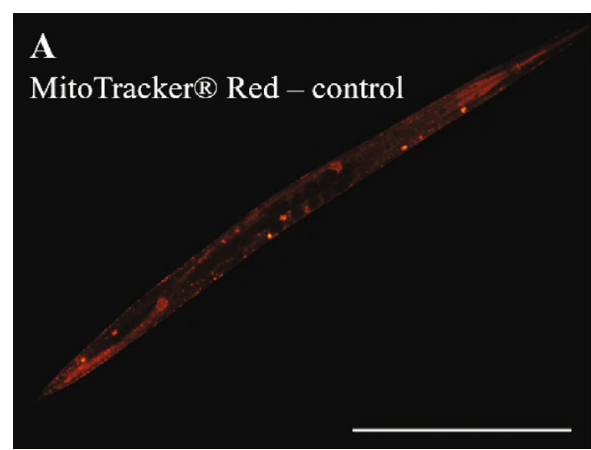

(a)

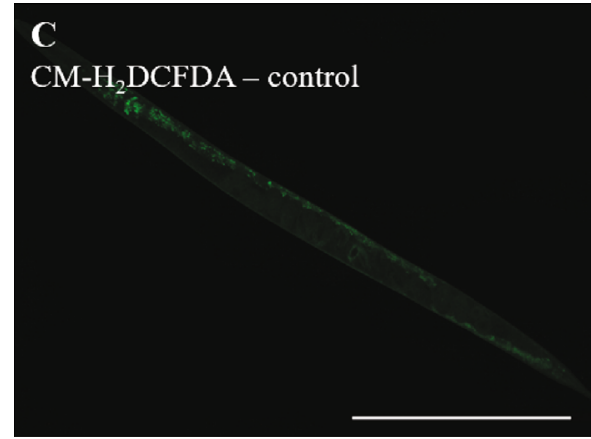

(c)

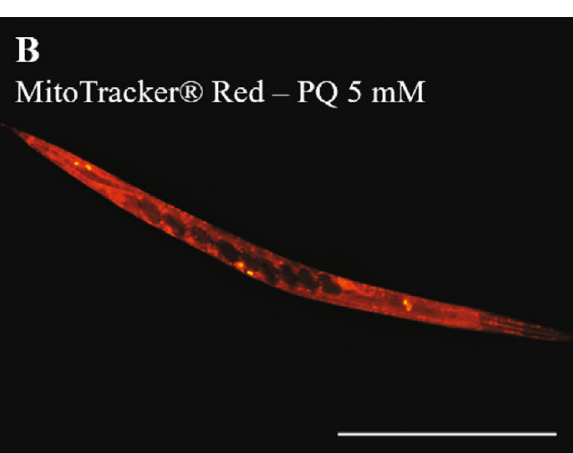

(b)

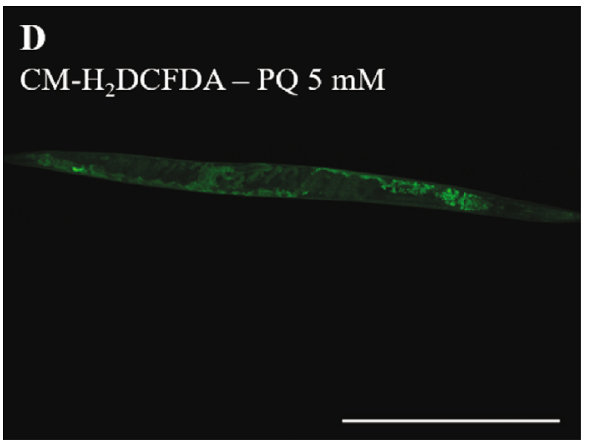

(d)

Figure 3: Nematodes, stained with MitoTracker ${ }^{\circledR}$ Red $(a, b)$ for mitochondrial and with CM-H ${ }_{2}$ DCFDA (c, d) for cytosolic site of ROS generation, treated for $4 \mathrm{~h}$ in the absence (control) or presence of $5 \mathrm{mM}$ PQ. Scaling bar is $400 \mu \mathrm{M}$.

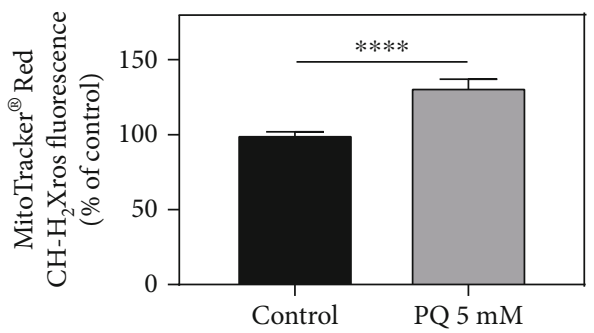

(a)

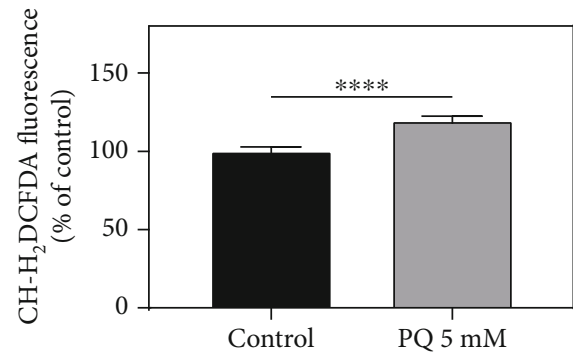

(b)

Figure 4: Paraquat (5 mM) increases mitochondrial and cytosolic ROS level in wild-type nematodes after $4 \mathrm{~h}$ of exposure. (a) MitoTracker ${ }^{\circledR}$ Red (CM- $\mathrm{H}_{2} \mathrm{Xros}$ ) was used to determine mitochondrial and (b) CM- $\mathrm{H}_{2}$ DCFDA for cytosolic ROS production. Mean \pm SEM; Student's $t$-test; ${ }^{* * * *} p<0.0001$.

mitochondrial respiratory chain and significantly reduced the activity of CI, CII, and CIV (Figure 7(c)).

The ratio between ETS and leak respiration after oligomycin addition, known as respiratory control ratio (RCR), is an accepted indicator of an increased proton gradient for ATP synthesis via complex V [37]. RCR was not altered after PQ incubation (data not shown).

The activity of citrate synthase (CS), as part of the Krebs cycle, is an established mitochondrial matrix marker [40]. PQ significantly reduced CS activity (Figure $7(\mathrm{~d})$ ) indicating a reduced mitochondrial mass [41].

3.6. PQ Decreases the Levels of Pyruvate and Lactate. Next, effects on energy metabolites were determined. Although
PQ significantly impaired $\Delta \Psi \mathrm{m}$, respiratory chain, and mitochondrial integrity, ATP levels were not significantly reduced (Figure 8(a)). The energetic metabolites pyruvate and lactate were significantly decreased (Figures 8(b) and 8(c)). The decline of lactate was stronger than the reduction of pyruvate, resulting in a significantly decreased lactate/pyruvate ratio after PQ treatment (Figure 8(d)), indicating a lower glycolytic turnover [42].

3.7. PQ Causes an Upregulation of Genes Relevant for Longevity and Mitochondrial Biogenesis. PQ affected the expression levels of several longevity- and mitochondrial biogenesis-related genes in C. elegans. The ortholog of human forkhead box $\mathrm{O} 1$ daf-16 known to be relevant for 


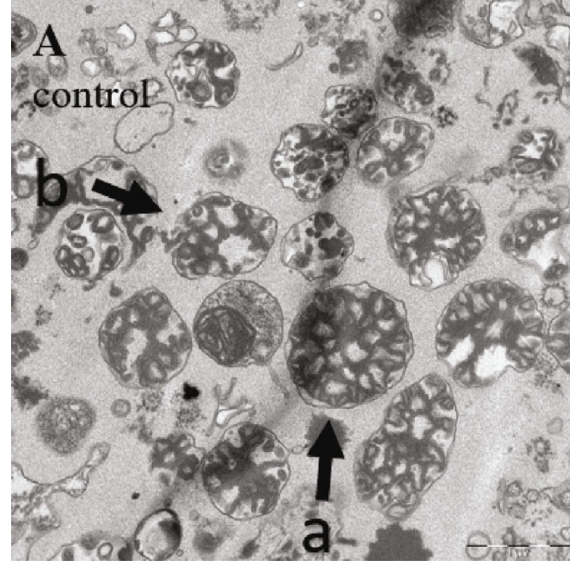

(a)

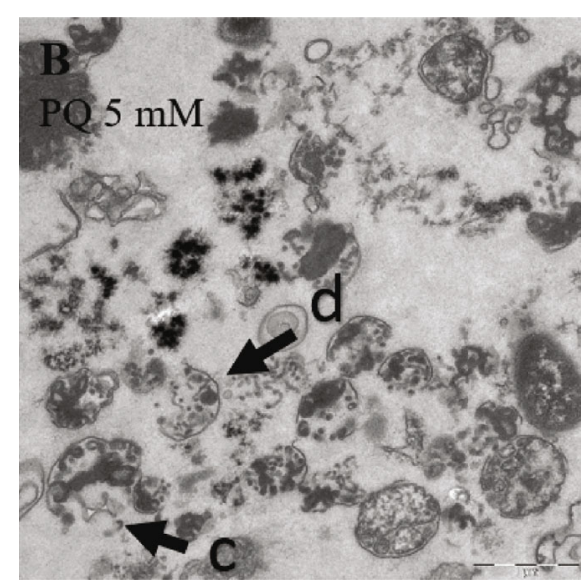

(b)

Figure 5: Transmission electron microscopic pictures of isolated mitochondria from C. elegans treated for $48 \mathrm{~h}$ in the absence (a; control) or presence of $5 \mathrm{mM}$ PQ (b). Degrees of damage are indicated by exemplary pointers (A= "intact"; $\mathrm{B}=$ "mildly fractured"; $\mathrm{C}=$ "heavily fractured"; and $\mathrm{D}=$ "fractured").

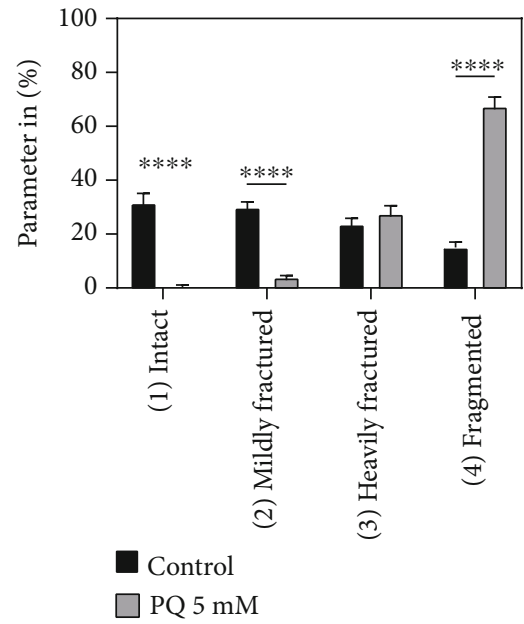

FIGURE 6: Mitochondria isolated from nematodes after a $48 \mathrm{~h}$ incubation in the absence (control) or presence of $5 \mathrm{mM}$ PQ were assessed by transmission electron microscopy and categorized into four categories (intact, mildly fractured, heavily fractured, and fragmented) as described in Material and Methods. Mean \pm SEM; Student's $t$-test; ${ }^{* * * *} p<0.0001$.

stress resistance, was insignificantly increased by $7 \%$, likewise to aak-2 (AMP-activated kinase) which was increased by $17 \%$ at its transcript level. Sir-2.1, which also plays a prominent role for longevity and stress-resistance and encodes an ortholog of human Sirtuin 1, was significantly increased by $73 \%$. Marker genes for mitochondrial biogenesis, i.e., skn-1, atfs-1, and atp-2, were all significantly upregulated at the mRNA level by paraquat (Figure 9).

\section{Discussion}

C. elegans represents a well-established model especially for investigations of longevity and genetic variations [43-46]. Mitochondria have been unravelled as organelles with a great impact on longevity and stress resistance [47]. While mitochondrial investigations in nematodes are conducted predominantly using fluorescent staining methods [48-50], mitochondrial isolation protocols are scarce [43]. In this study, we not only present data supporting the connection between mitochondrial function and longevity but also an easy and suitable way to isolate functional mitochondria. Questions concerned with the organelles' function are more distinct in a cell-free environment, free from intervening influences [28].

Paraquat (PQ) was used to induce oxidative stress resulting in mitochondrial dysfunction to display significant effects on the nematodes' lifespan and their respiratory chain capacity. PQ represents a known stress inducer impairing all respiratory chain complexes but most prominently complex I. In a reduced state, it uses oxygen as an oxidant producing the radical superoxide, mediating PQ toxicity [51]. PQ has been commonly used to trigger mitochondrial stress and dysfunction in C. elegans [52], as well as to reduce lifespan [53], and was therefore preferred over other known inducers of oxidative stress, as for example juglone, creating ROS through an increased apoptosis [54], or rotenone which specifically blocks complex I and therefore is commonly used for Parkinson's disease models [55].

Our goal was to identify links and causalities between mitochondrial dysfunction and a reduced lifespan and healthspan, as well as to establish a platform for future investigations concerned with potentially beneficial effects of nutrients or pharmaceuticals. We used a Balch Homogenizer and confirmed that the use of this technique represents a highly efficient and powerful tool to isolate mitochondrial fractions with a high quality [34].

4.1. Linking Longevity and Mitochondrial Function. In different experimental settings, various concentrations of PQ ranging from 0.2 to $25 \mathrm{mM}$ were used [56-59]. Thus, identifying an optimal concentration for our investigations was the initial step. In a heat-stress resistance assay, we tested PQ 


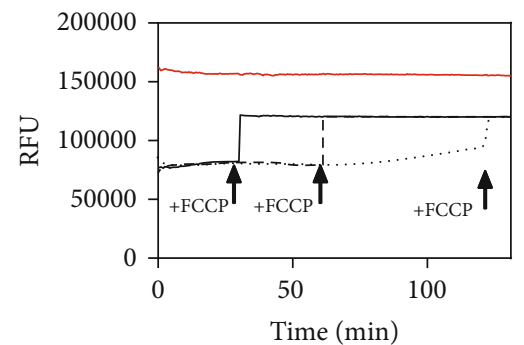

- Rh123

— Mitochondria+FCCP (30 min)

- - Mitochondria+FCCP (60 min)

..... Mitochondria+FCCP (120 min)

(a)

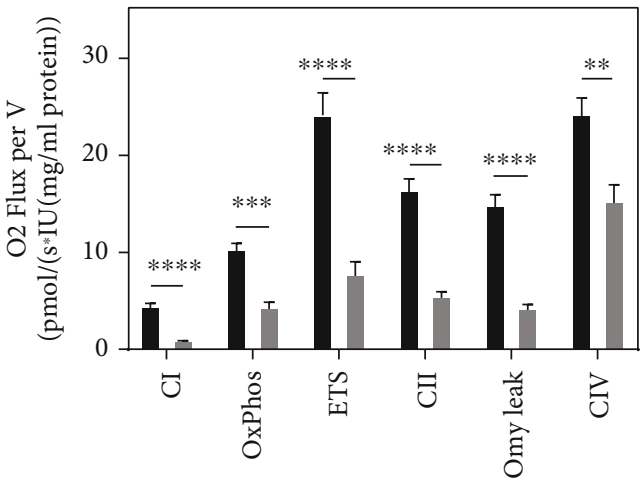

Pyruvate/malate

ADP

Succinate

FCPP

Rotenone

Oligomycin

Ascorbate/TMPD

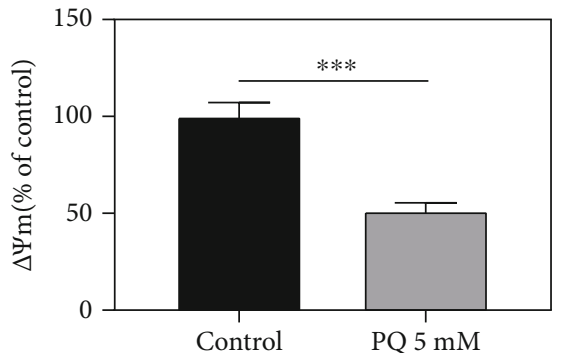

(b)

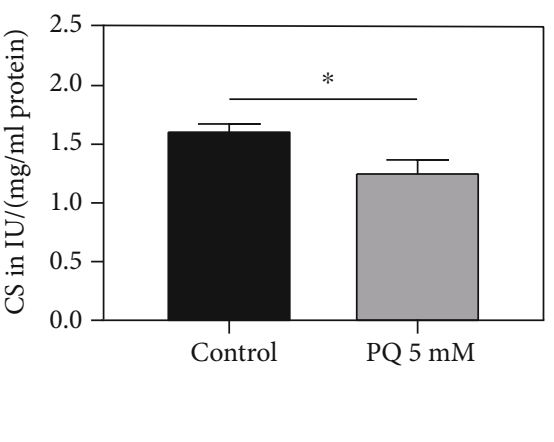

Control

$\square \mathrm{PQ} 5 \mathrm{mM}$

(c)

(d)

Figure 7: (a) Mitochondrial membrane potential $(\Delta \Psi \mathrm{m})$ over 130 minutes assessed by fluorescent dye rhodamine 123 (Rh123). Short circuit of $\Delta \Psi \mathrm{m}$ was achieved by the addition of FCCP after 30, 60, and 120 minutes. (b) $\Delta \Psi \mathrm{m}$ (MMP), assessed by mitochondria-dependent increase of Rh123 fluorescence in percentage, after PQ treatment $(5 \mathrm{mM})$ for $48 \mathrm{~h}$, detected with a ClarioStar Plate Reader (BMG, Ortenberg, Germany). (c) Respiration of isolated mitochondria from C. elegans normalized to (d) citrate synthase activity in international units $\mathrm{IU} /(\mathrm{mg} / \mathrm{mL}$ protein). Activity of respiration complexes was measured using an O2k Oxygraph (Oroboros, Innsbruck, Austria). Addition of substances into the Oxygraph's chambers is indicated with a plus sign $(+)$. Mean $\pm \mathrm{SEM}$; Student's $t$-test; ${ }^{*} p<0.05,{ }^{* *} p<0.01$, ${ }^{* * *} p<0.001$, and ${ }^{* * * *} p<0.0001$.

concentrations ranging from $0.01 \mathrm{mM}$ to $100 \mathrm{mM}$ to establish their effects on the nematodes' survival at $37^{\circ} \mathrm{C}$. A concentration-dependent decline in the nematodes' ability to tolerate heat stress of $37^{\circ} \mathrm{C}$ could be observed. In our experiments, a concentration of $5 \mathrm{mM}$ PQ was the lowest to reach a level of significance of ${ }^{* * *} p<0.0001$, lowering the median survival from $11 \mathrm{~h}$ to $8.5 \mathrm{~h}$ and was therefore selected for the following investigations. Interestingly, even though not significant, PQ at a concentration of $0.01 \mathrm{mM}$ led to a slightly increased tolerance of heat stress in wild-type nematodes. To validate PQ $5 \mathrm{mM}$, as a concentration high enough to alter the nematodes' lifespan, supposedly through mitochondrial stress, we tested this concentration in a survival experiment under physiological conditions. Again, PQ significantly decreased the nematodes' survival by $20 \%$ $\left({ }^{* *} p=0.0026\right)$ compared to control.

Similar to our results, Wu et al. used PQ as an oxidative stressor in an thermotolerance assay, resulting in a reduced survival time of nematodes [59]. While PQ is often used to generate oxidative stress [60], to our best knowledge, the effect of sole PQ exposure on nematode survival under physiological conditions has only been investigated with a 


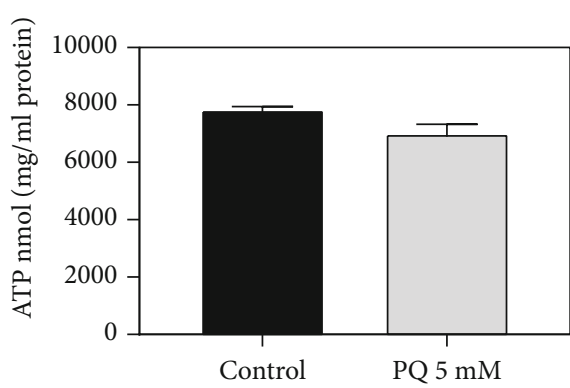

(a)

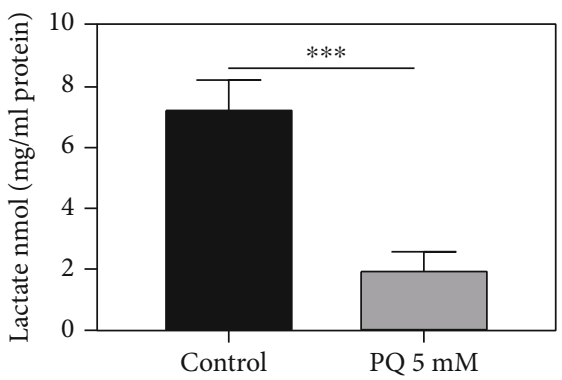

(c)

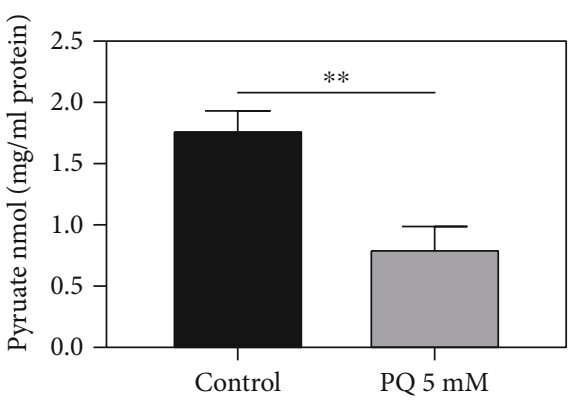

(b)

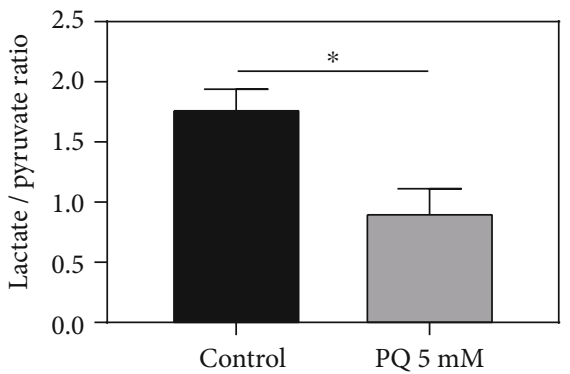

(d)

Figure 8: Determination of intracellular ATP levels (a), pyruvate (b), lactate (c), and lactate/pyruvate ratio (d) of wild-type C. elegans exposed to PQ $(5 \mathrm{mM})$ or not (control). ATP levels were assessed using an ATPlite luminescence assay, and lactate and pyruvate using two colorimetric assay kits. Values were normalized to protein concentrations. $n=8$; Mean \pm SEM; Student's $t$-test; ${ }^{*} p<0.05$, ${ }^{* *} p<0.01$, and *** $p<0.001$.

concentration of $0.25 \mathrm{mM}$ [58]. This low-dose exposure resulted in an extended lifespan, comparable to our heatstress survival assay, showing a slightly increased stress resistance after $0.01 \mathrm{mM}$ PQ exposure, suggesting a mitohormetic effect $[61,62]$. Generally, a close relation between elevated reactive oxygen species and shortened lifespan expectancy can be drawn throughout investigations and species [63, 64], which is in line with our findings.

Nematodes retrive their food through chemotaxis $[33,65]$. Mitochondrial dysfunction not only effects longevity as such but also impairs neuronal function and consequently the animals' sensory ability of tracking food. This closely links chemotaxis and longevity through cellular and neuronal impairment caused by oxidative stress [66]. PQ significantly decreased chemotaxis in C. elegans, thus further strengthening the connection between mitochondrial dysfunction and longevity from another point of view. To our best knowledge, the effect of PQ on chemotaxis has never been invested but fits our previous findings and is coherent with the results of $\mathrm{Wu}$ et al. showing chemotaxis deficits caused through $\mathrm{A} \beta$-mediated oxidative stress [67].

\subsection{Isolation and Qualification of Isolated Nematode} Mitochondria. Mitochondrial investigations in C. elegans so far have mostly been restricted to measuring overall oxygen consumption, using, for example, an O2k Oxygraph from Oroboros, or fluorescent staining of whole cells or nematodes targeting ROS generation or membrane potentials [68-71]. Studies focusing on isolated mitochondria, however, have been scarce $[43,71]$. Isolated mitochondria have distinct advantages compared to whole cell or organism systems, since they allow investigations free from interfering organelles or cellular reactions and make it possible to draw direct causalities $[27,28]$. A challenge is the nematodes' resilient cuticle making it hard to gather sufficient and especially efficient mitochondria. Several techniques have been described, ranging from grinding worms with a pestle after snap freezing in liquid nitrogen, "bead beating" with glass beads, to mechanically disrupting the nematodes' cuticle, or sonication. Nonetheless, each method suffers from one or more limitations. Grinding requires relatively large sample sizes, which are easily lost [72]. When applying "bead beating" or sonication, samples are prone to denaturation as samples heat up during the process and potentially damaging mitochondria [73]. Furthermore, sonication is unable to produce intact nucleic acids.

Balch homogenization is able to effectively break C. elegans cuticles of any stages [72]. The system is able to produce functional proteins, qRT-PCR quality mRNA, and intact mitochondria of a high quality and intactness. Through changeable ball clearance sizes, or number of syringe passes, homogenization roughness can be controlled [34, 74].

Mitochondria isolated with a $12 \mu \mathrm{m}$ ball clearance appear more intact, after electron microscopy, compared to isolates gathered with a ball clearance leaving only a $6 \mu \mathrm{m}$ gap (see Supp. 1). A semiquantitative and double-blinded counting validates the first impression and shows that especially percentage numbers in the "heavily fractured" (32\%) and "fragmented" (25\%) categories are higher for mitochondria isolated with a $6 \mu \mathrm{m}$ ball clearance, compared to $12 \mu \mathrm{m}$. Here, 

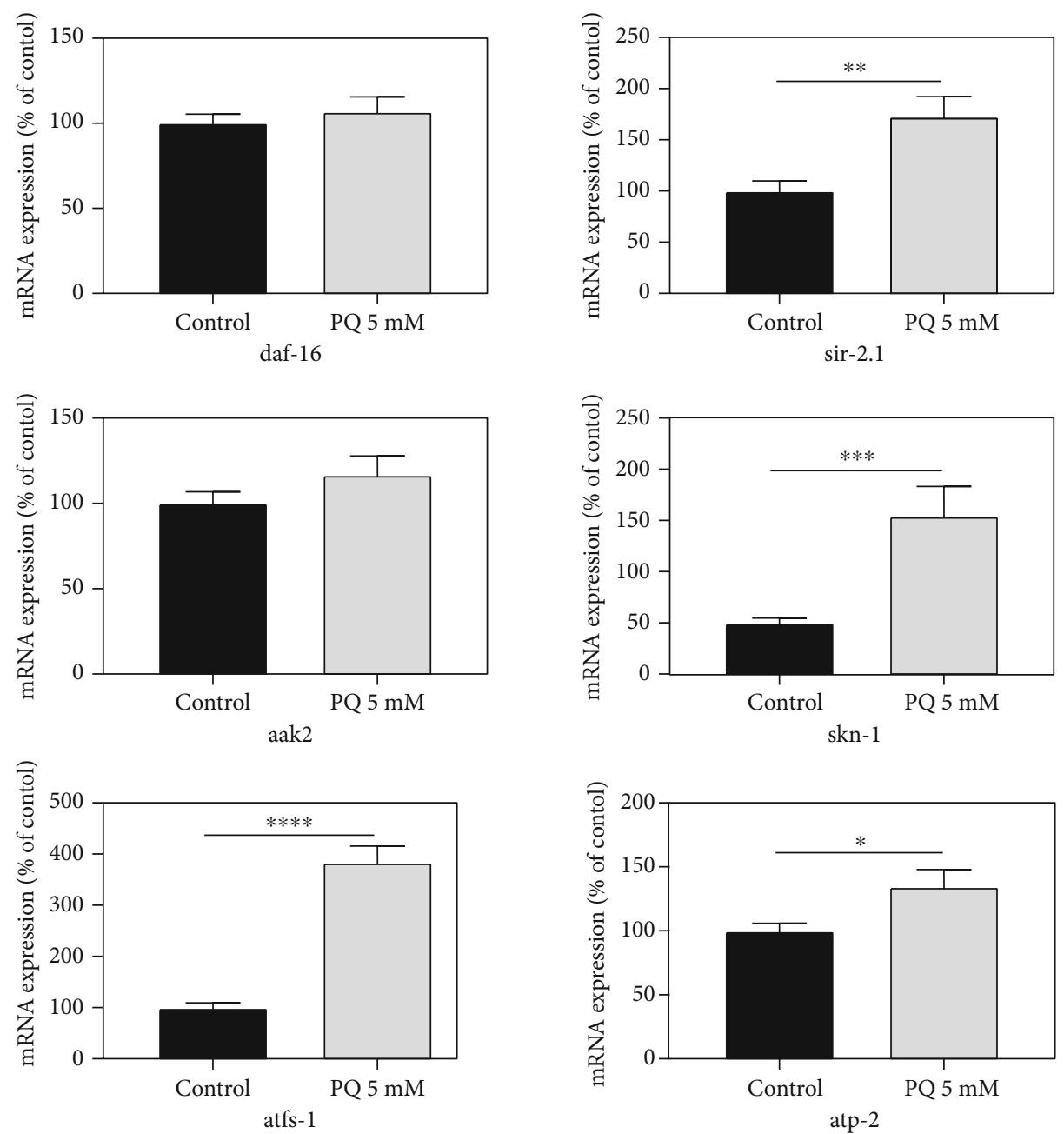

FiguRE 9: Relative normalized mRNA expression levels of longevity- and mitochondrial biogenesis-related genes daf-16, sir-2.1, aak-2, skn-1, atfs- 1 , and atp- 2 in C. elegans in the absence or presence of PQ (5 mM); Mean \pm SEM; Student's $t$-test; ${ }^{*} p<0.05,{ }^{* *} p<0.01,{ }^{* * *} p<0.001$, and ${ }^{* * * *} p<0.0001$; Results are normalized to the mRNA expression levels of amanitin resistant (ama-1) and actin (act-2).

only $24 \%$ was "heavily fractured" and $15 \%$ fragmented. Rougher homogenization using a $6 \mu \mathrm{m}$ clearance results in only $15 \%$ fully intact mitochondria compared to $32 \%$ for a $12 \mu \mathrm{m}$ clearance (see Supp. 2).

The applicability of isolation of mitochondria with a $12 \mu \mathrm{m}$ clearance was further evidenced by addition of cytochrome $c$ which would enter, if fractured, the outer mitochondrial membrane resulting in an increased respiration rate [75]. No changes of respiration, however, could be observed after isolation with a $12 \mu \mathrm{m}$ clearance, supporting the integrity of the outer membrane, whereas a significant increase in respirational flux was measured after isolation with a $6 \mu \mathrm{m}$ clearance (see Supp. 3).

Since none of our described experimental procedures takes more than 30 minutes after isolation, mitochondrial stability over that time period needed to be ensured. We could demonstrate a stable $\Delta \Psi$ m over more than 60 minutes giving more than twice the time needed for all the experiments described. Furthermore, this verifies the $12 \mu \mathrm{m}$ ball clearance and 5 strokes applied, as valid parameters to generate a sufficient and intact mitochondrial fraction, as evidenced by transmission electron microscopy. Treatment of nematodes with $5 \mathrm{mM}$ PQ, however, caused a significant increase in heavily fractured and fragmented mitochondria characterized by membrane rupture and damaged crista structure.

4.3. Impact of Paraquat on Mitochondrial Function. Paraquat at a concentration of $5 \mathrm{mM}$ was identified to reduce significantly the stress resistance as well as longevity in wild-type nematodes. This was associated with a significantly reduced mitochondrial membrane potential $(\Delta \Psi \mathrm{m})$ after $48 \mathrm{~h}$. A similar decrease in $\Delta \Psi \mathrm{m}$, after PQ treatment, was observed by Zhang et al. and $\mathrm{Wu}$ et al., while the latter also described a decreased expression of complex I, II, and III genes of the mitochondrial respiratory chain (ETC) $[76,77]$. The latter findings are in line with the results of our respiration experiments, where PQ significantly decreased respiration rates of complex I and II. A decreased activity could be observed in our experiments also for the respiratory complexes CI and CII as well as for the OxPhos, ETS, and OmyLeak. Interestingly, complex IV was not altered in its activity. Citrate 
synthase activity (CS) represents an established indicator for the mitochondrial mass [40]. Thus, the decreased CS activity in our experiments indicates that PQ reduced the mitochondrial mass. These results fit to reports describing a reduced respiratory capacity and a reduced mitochondrial mass after $4 \mathrm{mM}$ PQ exposure in a PC12 cell model [78] or decreased respiration rates in zebra fish [79].

To validate an increased ROS generation by PQ as the reason for mitochondrial dysfunction, we investigated mitochondrial and also cytosolic ROS levels. Since PQ treatment for $48 \mathrm{~h}$ decreased intestinal pumping of nematodes causing an insufficient uptake of both fluorescent markers (data not shown), we had to decrease the time of exposure to $4 \mathrm{~h}$. Nevertheless, also after this short incubation period, ROS levels at both locations were significantly increased which is in line with previous findings $[14,58,59,80,81]$.

According to the observed dysfunction of mitochondria, it appeared reasonable to suggest that PQ causes limitations in the generation of ATP and by the accumulation of substrates also reduces the glycolytic flux. Interestingly, only slightly decreased ATP concentrations were found. This might be explained by the increased ATP synthase activity as a consequence of enhanced atp- 2 mRNA levels observed here. Significant ATP depletion of approximately 20\% was found, however, at higher concentrations of $10 \mathrm{mM}$ PQ in nematodes [76]. Pyruvate and lactate levels, however, were significantly decreased after PQ insult in our studies. Similar results have already been shown for glycolytic metabolites after PQ exposure in SK-N-SH cells [82].

To elaborate genetic pathways affected by PQ exposure, we assessed the mRNA levels of $d a f-16$, aak-2, and sir-2.1, three genes with relevance for longevity and stressresistance longevity $[70,77,78]$. While $d a f-16$ and aak-2 were only slightly elevated due to PQ, expression of the human Sirtuin 1 ortholog sir-2.1 was significantly increased by $73 \%$. Daf-16 as key modulator of longevity in C. elegans, is activated, amongst others, by sir-2.1 and aak-2 [83]. The lack of enhanced expression of daf-16 lets us suggest that the enhanced expression of sir-2.1 is not sufficient to activate daf-16 expression in the presence of PQ. However, another gene important for stress resistance and also for mitogenesis, which was found to be significantly upregulated, is the $n r f-2$ homolog $s k n-1$. Skn-1 upregulation was also found to increase the activity of PGC1 $\alpha$, the mammalian key regulator of mitogenesis, which, however, has no direct homolog in the nematode $[50,84]$. Adaptations to compensate for the PQ-induced stress are evident finally also by enhanced expression of atfs-1, a key regulator of mitochondrial unfolded protein response $\left(\mathrm{UPR}^{\mathrm{mt}}\right)$.

\section{Conclusions}

Using paraquat as a stressor, we here describe the close association of oxidative stress, restricted energy metabolism, reduced stress resistance, and longevity in the nematode Caenorhabditis elegans, focusing on isolated mitochondria, making it a readily accessible in vivo model for mitochondrial dysfunction.

\section{Data Availability}

The dataset generated during this study is available from the corresponding author on reasonable request.

\section{Conflicts of Interest}

The authors declare that they have no conflict of interest.

\section{Acknowledgments}

The help of Hans Zischka and Sabine Schmitt (Helmholz Zentrum, Munich) for introducing us to the described Balch Homogenizer, showing us how to use it properly, and introducing us into the field of transmission electron microscopy is highly appreciated. This work is supported by FlexiFond "Mitochondrial-Network" (number 2018_1_1_2).

\section{Supplementary Materials}

Supp.1: transmission electron microscopic pictures of mitochondria from C. elegans isolated with a $12 \mu \mathrm{m}$ (A) or $6 \mu \mathrm{m}$ ball clearance (B). Degrees of damage are indicated by exemplary pointers ( $\mathrm{a}=$ "intact"; $\mathrm{b}=$ "mildly fractured"; $c=$ "heavily fractured"; and $d=$ "fractured"). Supp. 2: mitochondria isolated from nematodes with $12 \mu \mathrm{m}$ or $6 \mu \mathrm{m}$ clearance were assessed by transmission electron microscopy and categorized into four categories (intact, mildly fractured, heavily fractured, and fragmented) as described in Material and Methods. Isolation with $6 \mu \mathrm{m}$ ball clearance resulted in significantly less intact as well as more fragmented mitochondria. Mean \pm SEM; Student's $t$-test; ${ }^{*} p<0.05,{ }^{* *} p<0.01$. Supp. 3: cytochrome $c$ addition, as a marker for intactness of the outer mitochondrial membrane, increases respiration after isolation with a $6 \mu \mathrm{m}$ compared to a $12 \mu \mathrm{m}$ clearance, indicating a fractured outer membrane. $n=5$; Mean \pm SEM; Students $t$-test; ${ }^{* * *} p<0.001$. (Supplementary Materials)

\section{References}

[1] P.-P. Tan, B.-H. Zhou, W.-P. Zhao, L. S. Jia, J. Liu, and H. W. Wang, "Mitochondria-mediated pathway regulates $\mathrm{C} 2 \mathrm{C} 12$ cell apoptosis induced by fluoride," Biological Trace Element Research, vol. 185, no. 2, pp. 440-447, 2018.

[2] J. L. Aguilar-Lopez, R. Laboy, F. Jaimes-Miranda, E. Garay, A. DeLuna, and S. Funes, "Slm35 links mitochondrial stress response and longevity through TOR signaling pathway," Aging, vol. 8, no. 12, pp. 3255-3271, 2016.

[3] A. Raffaello and R. Rizzuto, "Mitochondrial longevity pathways," Biochimica et Biophysica Acta, vol. 1813, no. 1, pp. 260-268, 2011.

[4] T. Finkel, "The metabolic regulation of aging," Nature Medicine, vol. 21, no. 12, pp. 1416-1423, 2015.

[5] H.-W. Chang, S. Pisano, A. Chaturbedi et al., "Transcription factors CEP-1/p53 and CEH-23 collaborate with AAK2/AMPK to modulate longevity in Caenorhabditis elegans.," Aging Cell, vol. 16, no. 4, pp. 814-824, 2017.

[6] Y. Wang and S. Hekimi, "Mitochondrial dysfunction and longevity in animals: untangling the knot," Science, vol. 350, no. 6265, pp. 1204-1207, 2015. 
[7] B. Dilberger, M. Passon, H. Asseburg et al., "Polyphenols and metabolites enhance survival in rodents and nematodesimpact of mitochondria," Nutrients, vol. 11, no. 8, p. 1886, 2019.

[8] D. Harman, "Aging: a theory based on free radical and radiation chemistry," Journal of Gerontology, vol. 11, no. 3, pp. 298-300, 1956.

[9] A. Y. Seo, A. M. Joseph, D. Dutta, J. C. Y. Hwang, J. P. Aris, and C. Leeuwenburgh, "New insights into the role of mitochondria in aging: mitochondrial dynamics and more," Journal of Cell Science, vol. 123, no. 15, pp. 2533-2542, 2010.

[10] D. Harman, "The biologic clock: the mitochondria?," Journal of the American Geriatrics Society, vol. 20, no. 4, pp. 145147, 1972.

[11] R. S. Balaban, S. Nemoto, and T. Finkel, "Mitochondria, oxidants, and aging," Cell, vol. 120, no. 4, pp. 483-495, 2005.

[12] W. Yang and S. Hekimi, "A mitochondrial superoxide signal triggers increased longevity in Caenorhabditis elegans," PLoS Biology, vol. 8, no. 12, p. e1000556, 2010.

[13] J. M. Copeland, J. Cho, T. Lo Jr. et al., "Extension of_Drosophila_ Life Span by RNAi of the Mitochondrial Respiratory Chain," Current Biology, vol. 19, no. 19, pp. 1591-1598, 2009.

[14] S. W. Smith, L. C. Latta, D. R. Denver, and S. Estes, "Endogenous ROS levels in C. elegans under exogenous stress support revision of oxidative stress theory of life-history tradeoffs," BMC Evolutionary Biology, vol. 14, no. 1, p. 161, 2014.

[15] A. Dillin, A. L. Hsu, N. Arantes-Oliveira et al., "Rates of behavior and aging specified by mitochondrial function during development," Science, vol. 298, no. 5602, pp. 2398-2401, 2002.

[16] P. A. Kirchman, S. Kim, C. Y. Lai, and S. M. Jazwinski, "Interorganelle signaling is a determinant of longevity in Saccharomyces cerevisiae," Genetics, vol. 152, no. 1, pp. 179-190, 1999.

[17] S. S. Lee, R. Y. N. Lee, A. G. Fraser, R. S. Kamath, J. Ahringer, and G. Ruvkun, "A systematic RNAi screen identifies a critical role for mitochondria in _C. elegans_ longevity," Nature Genetics, vol. 33, no. 1, pp. 40-48, 2003.

[18] X. Liu, N. Jiang, B. Hughes, E. Bigras, E. Shoubridge, and S. Hekimi, "Evolutionary conservation of the clk-1dependent mechanism of longevity: loss of mclk1 increases cellular fitness and lifespan in mice," Genes \& Development, vol. 19, no. 20, pp. 2424-2434, 2005.

[19] W. B. Wood, The Nematode Caenorhabditis Elegans, Cold Spring Harbor Laboratory, Cold Spring Harbor, N.Y, 1988.

[20] J. Gruber, C. B. Chen, S. Fong, L. F. Ng, E. Teo, and B. Halliwell, "Caenorhabditis elegans: what we can and cannot learn from aging worms," Antioxidants \& Redox Signaling, vol. 23, no. 3, pp. 256-279, 2015.

[21] S. Schmeisser, K. Schmeisser, S. Weimer et al., "Mitochondrial hormesis links low-dose arsenite exposure to lifespan extension," Aging Cell, vol. 12, no. 3, pp. 508-517, 2013.

[22] K. Burkewitz, Y. Zhang, and W. B. Mair, "AMPK at the nexus of energetics and aging," Cell Metabolism, vol. 20, no. 1, pp. 10-25, 2014.

[23] A. M. Nargund, M. W. Pellegrino, C. J. Fiorese, B. M. Baker, and C. M. Haynes, "Mitochondrial import efficiency of ATFS-1 regulates mitochondrial UPR activation," Science, vol. 337, no. 6094, pp. 587-590, 2012.

[24] T. J. Schulz, K. Zarse, A. Voigt, N. Urban, M. Birringer, and M. Ristow, "Glucose restriction extends _Caenorhabditis elegans_ life span by inducing mitochondrial respiration and increasing oxidative stress," Cell Metabolism, vol. 6, no. 4, pp. 280-293, 2007.

[25] N. Ventura, S. L. Rea, A. Schiavi, A. Torgovnick, R. Testi, and T. E. Johnson, "p53/CEP-1 increases or decreases lifespan, depending on level of mitochondrial bioenergetic stress," Aging Cell, vol. 8, no. 4, pp. 380-393, 2009.

[26] L. Walter, A. Baruah, H. W. Chang, H. M. Pace, and S. S. Lee, "The homeobox protein CEH-23 mediates prolonged longevity in response to impaired mitochondrial electron transport chain in C. elegans," PLoS Biology, vol. 9, no. 6, p. e1001084, 2011.

[27] C. Frezza, S. Cipolat, and L. Scorrano, "Organelle isolation: functional mitochondria from mouse liver, muscle and cultured filroblasts," Nature Protocols, vol. 2, no. 2, pp. 287-295, 2007.

[28] K.-M. Debatin, D. Poncet, and G. Kroemer, "Chemotherapy: targeting the mitochondrial cell death pathway," Oncogene, vol. 21, no. 57, pp. 8786-8803, 2002.

[29] S. Brenner, "The genetics of Caenorhabditis elegans," Genetics, vol. 77, no. 1, pp. 71-94, 1974.

[30] T. Stiernagle, "Maintenance of C. elegans," C. elegans, vol. 2, pp. 51-67, 1999.

[31] F. R. G. Amrit, R. Ratnappan, S. A. Keith, and A. Ghazi, "The _C. elegans_lifespan assay toolkit," Methods, vol. 68, no. 3, pp. $465-475,2014$.

[32] E. Fitzenberger, D. J. Deusing, C. Marx, M. Boll, K. Lüersen, and U. Wenzel, "The polyphenol quercetin protects the mev1 mutant of Caenorhabditis elegans from glucose-induced reduction of survival under heat-stress depending on SIR-2.1, DAF-12, and proteasomal activity," Molecular Nutrition \& Food Research, vol. 58, no. 5, pp. 984-994, 2014.

[33] O. Margie, C. Palmer, and I. Chin-Sang, "C. elegans chemotaxis assay," JoVE (Journal of Visualized Experiments), vol. 74, article e50069, 2013.

[34] S. Schmitt, F. Saathoff, L. Meissner et al., "A semi-automated method for isolating functionally intact mitochondria from cultured cells and tissue biopsies," Analytical Biochemistry, vol. 443, no. 1, pp. 66-74, 2013.

[35] W. E. Balch and J. E. Rothman, "Characterization of protein transport between successive compartments of the Golgi apparatus: asymmetric properties of donor and acceptor activities in a cell-free system," Archives of Biochemistry and Biophysics, vol. 240, no. 1, pp. 413-425, 1985.

[36] S. Stadlmann, K. Renner, J. Pollheimer et al., "Preserved coupling of oxidative phosphorylation but decreased mitochondrial respiratory capacity in IL-1beta-treated human peritoneal mesothelial cells," Cell Biochemistry and Biophysics, vol. 44, no. 2, pp. 179-186, 2006.

[37] S. Hagl, A. Kocher, C. Schiborr et al., "Rice bran extract protects from mitochondrial dysfunction in guinea pig brains," Pharmacological Research, vol. 76, pp. 17-27, 2013.

[38] E. Hutter, K. Renner, G. Pfister, P. Stöckl, P. Jansen-Dürr, and E. Gnaiger, "Senescence-associated changes in respiration and oxidative phosphorylation in primary human fibroblasts," The Biochemical Journal, vol. 380, pp. 919-928, 2004.

[39] A. V. Kuznetsov, D. Strobl, E. Ruttmann, A. Königsrainer, R. Margreiter, and E. Gnaiger, "Evaluation of mitochondrial respiratory function in small biopsies of liver," Analytical Biochemistry, vol. 305, no. 2, pp. 186-194, 2002.

[40] S. Larsen, J. Nielsen, C. N. Hansen et al., "Biomarkers of mitochondrial content in skeletal muscle of healthy young human 
subjects," The Journal of Physiology, vol. 590, no. 14, pp. 33493360, 2012.

[41] R. Rabøl, S. Larsen, P. M. V. Højberg et al., "Regional anatomic differences in skeletal muscle mitochondrial respiration in type 2 diabetes and obesity," The Journal of Clinical Endocrinology and Metabolism, vol. 95, no. 2, pp. 857-863, 2010.

[42] S. Yanase, H. Suda, K. Yasuda, and N. Ishii, "Impaired p53/CEP-1 is associated with lifespan extension through an age-related imbalance in the energy metabolism of C. elegans," Genes to Cells, vol. 22, no. 12, pp. 1004-1010, 2017.

[43] N. Castelein, M. Muschol, I. Dhondt et al., "Mitochondrial efficiency is increased in axenically cultured _Caenorhabditis elegans_," Experimental Gerontology, vol. 56, pp. 26-36, 2014.

[44] E. Fitzenberger, D. J. Deusing, A. Wittkop et al., "Effects of plant extracts on the reversal of glucose-induced impairment of stress-resistance in Caenorhabditis elegans," Plant Foods for Human Nutrition, vol. 69, no. 1, pp. 78-84, 2014.

[45] H. J. Weir, P. Yao, F. K. Huynh et al., "Dietary restriction and AMPK increase lifespan via mitochondrial network and peroxisome remodeling," Cell Metabolism, vol. 26, no. 6, pp. 884-896.e5, 2017.

[46] The C elegans Sequencing Consortium, "Genome sequence of the nematode C. elegans: a platform for investigating biology," Science, vol. 282, no. 5, pp. 2012-2018, 1998.

[47] S. Tan and E. Wong, "Mitophagy transcriptome: mechanistic insights into polyphenol-mediated mitophagy," Oxidative Medicine and Cellular Longevity, vol. 2017, Article ID 9028435, 2017.

[48] E. F. Fang, T. B. Waltz, H. Kassahun et al., "Tomatidine enhances lifespan and healthspan in C. elegans through mitophagy induction via the SKN-1/Nrf2 pathway," Scientific reports, vol. 7, 2017.

[49] T. Heidler, K. Hartwig, H. Daniel, and U. Wenzel, "Caenorhabditis elegans lifespan extension caused by treatment with an orally active ROS-generator is dependent on DAF-16 and SIR-2.1," Biogerontology, vol. 11, no. 2, pp. 183-195, 2010.

[50] K. Palikaras, E. Lionaki, and N. Tavernarakis, "Coordination of mitophagy and mitochondrial biogenesis during ageing in _C. elegans_," Nature, vol. 521, no. 7553, pp. 525-528, 2015.

[51] H. M. Cocheme and M. P. Murphy, "Complex I is the major site of mitochondrial superoxide production by paraquat," The Journal of Biological Chemistry, vol. 283, no. 4, pp. 1786-1798, 2008.

[52] R. J. Mockett, A. C. V. Bayne, L. K. Kwong, W. C. Orr, and R. S. Sohal, "Ectopic expression of catalase in _Drosophila_ mitochondria increases stress resistance but not longevity," Free Radical Biology \& Medicine, vol. 34, no. 2, pp. 207-217, 2003.

[53] J. N. Sampayo, A. Olsen, and G. J. Lithgow, "Oxidative stress in Caenorhabditis elegans: protective effects of superoxide dismutase/catalase mimetics," Aging Cell, vol. 2, no. 6, pp. 319-326, 2003.

[54] J. Wu, H. Zhang, Y. Xu et al., "Juglone induces apoptosis of tumor stem-like cells through ROS-p38 pathway in glioblastoma," BMC Neurology, vol. 17, no. 1, 2017.

[55] M. Maulik, S. Mitra, A. Bult-Ito, B. E. Taylor, and E. M. Vayndorf, "Behavioral phenotyping and pathological indicators of Parkinson's disease in C. elegans models," Frontiers in Genetics, vol. 8, 2017.

[56] M. E. Lima, A. C. Colpo, W. G. Salgueiro, G. E. Sardinha, D. S. Avila, and V. Folmer, "Ilex paraguariensis extract increases lifespan and protects against the toxic effects caused by para- quat in Caenorhabditis elegans," International Journal of Environmental Research and Public Health, vol. 11, no. 10, pp. 10091-10104, 2014.

[57] M. Fujii, N. Tanaka, K. Miki, M. N. Hossain, M. Endoh, and D. Ayusawa, "Uncoupling of longevity and paraquat resistance in mutants of the NematodeCaenorhabditis elegans," Bioscience, Biotechnology, and Biochemistry, vol. 69, no. 10, pp. 2015-2018, 2005.

[58] A. B. Hwang, E.-A. Ryu, M. Artan et al., "Feedback regulation via AMPK and HIF-1 mediates ROS-dependent longevity in Caenorhabditis elegans," Proceedings of the National Academy of Sciences of the United States of America, vol. 111, no. 42, pp. E4458-E4467, 2014.

[59] M. Wu, X. Kang, Q. Wang, C. Zhou, C. Mohan, and A. Peng, "Regulator of $G$ protein signaling-1 modulates paraquatinduced oxidative stress and longevity via the insulin like signaling pathway in _Caenorhabditis elegans_, Toxicology Letters, vol. 273, pp. 97-105, 2017.

[60] S.-I. Oh, J.-K. Park, and S.-K. Park, "Lifespan extension and increased resistance to environmental stressors by $\mathrm{N}$-acetylL-cysteine in Caenorhabditis elegans," Clinics, vol. 70, no. 5, pp. 380-386, 2015.

[61] E. J. Calabrese, V. Calabrese, and J. Giordano, "The role of hormesis in the functional performance and protection of neural systems," Brain Circulation, vol. 3, no. 1, pp. 1-13, 2017.

[62] V. Calabrese, C. Cornelius, A. T. Dinkova-Kostova, E. J. Calabrese, and M. P. Mattson, "Cellular stress responses, the hormesis paradigm, and vitagenes: novel targets for therapeutic intervention in neurodegenerative disorders," Antioxidants \& Redox Signaling, vol. 13, no. 11, pp. 1763-1811, 2010.

[63] A. Vikram, R. Anish, A. Kumar, D. N. Tripathi, and R. K. Kaundal, "Oxidative stress and autophagy in metabolism and longevity," Oxidative Medicine and Cellular Longevity, vol. 2017, Article ID 3451528, 3 pages, 2017.

[64] V. Marasco, A. Stier, W. Boner, K. Griffiths, B. Heidinger, and P. Monaghan, "Environmental conditions can modulate the links among oxidative stress, age, and longevity," Mechanisms of Ageing and Development, vol. 164, pp. 100-107, 2017.

[65] C. I. Bargmann, "Chemosensation in C. elegans," in WormBook: The Online Review of C. elegans Biology, WormBook, 2006.

[66] S. Maglioni, A. Schiavi, A. Runci, A. Shaik, and N. Ventura, "Mitochondrial stress extends lifespan in_C. elegans_through neuronal hormesis," Experimental Gerontology, vol. 56, pp. 89-98, 2014.

[67] Y. Wu, Z. Wu, P. Butko et al., "Amyloid-beta-induced pathological behaviors are suppressed by Ginkgo biloba extract EGb 761 and ginkgolides in transgenic Caenorhabditis elegans," The Journal of neuroscience : the official journal of the Society for Neuroscience, vol. 26, no. 50, pp. 13102-13113, 2006.

[68] Y. J. Kwon, S. Guha, F. Tuluc, and M. J. Falk, "High-throughput BioSorter quantification of relative mitochondrial content and membrane potential in living _Caenorhabditis elegans_," Mitochondrion, vol. 40, pp. 42-50, 2018.

[69] B. P. Braeckman, A. Smolders, P. Back, and S. de Henau, "In vivo detection of reactive oxygen species and redox status in Caenorhabditis elegans," Antioxidants \& Redox Signaling, vol. 25, no. 10, pp. 577-592, 2016.

[70] P. Gubert, B. Puntel, T. Lehmen et al., "Metabolic effects of manganese in the nematode _Caenorhabditis elegans_ 
through DAergic pathway and transcription factors activation," Neurotoxicology, vol. 67, pp. 65-72, 2018.

[71] J. R. Daniele, K. Heydari, E. A. Arriaga, and A. Dillin, "Identification and characterization of mitochondrial subtypes in Caenorhabditis elegans via analysis of individual mitochondria by flow cytometry," Analytical Chemistry, vol. 88, no. 12, pp. 6309-6316, 2016.

[72] S. Bhaskaran, J. A. Butler, S. Becerra, V. Fassio, M. Girotti, and S. L. Rea, "Breaking_Caenorhabditis elegans_the easy way using the Balch homogenizer: an old tool for a new application," Analytical Biochemistry, vol. 413, no. 2, pp. 123-132, 2011.

[73] N. J. Szewczyk, R. L. Mancinelli, W. McLamb, D. Reed, B. S. Blumberg, and C. A. Conley, "Caenorhabditis elegans survives atmospheric breakup of STS-107, space shuttle Columbia," Astrobiology, vol. 5, no. 6, pp. 690-705, 2005.

[74] S. Schmitt, C. Eberhagen, S. Weber, M. Aichler, and H. Zischka, "Isolation of mitochondria from cultured cells and liver tissue biopsies for molecular and biochemical analyses," Methods in Molecular Biology, vol. 1295, pp. 87-97, 2015.

[75] M. Wikstrom and R. Casey, "The oxidation of exogenous cytochrome c by mitochondria," FEBS Letters, vol. 183, no. 2, pp. 293-298, 1985.

[76] J. Zhang, R. Shi, H. Li et al., "Antioxidant and neuroprotective effects of_Dictyophora indusiata_polysaccharide in_Caenorhabditis elegans_," Journal of Ethnopharmacology, vol. 192, pp. 413-422, 2016.

[77] S. Wu, L. Lei, Y. Song et al., "Mutation of_hop-1_ and _pink1 _ attenuates vulnerability of neurotoxicity in _C. elegans_ : the role of mitochondria-associated membrane proteins in Parkinsonism," Experimental Neurology, vol. 309, pp. 67-78, 2018.

[78] Q. Zhou, H. Zhang, Q. Wu, J. Shi, and S. Zhou, "Pharmacological manipulations of autophagy modulate paraquat-induced cytotoxicity in PC12 cells," International Journal of Biochemistry and Molecular Biology, vol. 8, no. 2, pp. 13-22, 2017.

[79] X. H. Wang, C. L. Souders, Y. H. Zhao, and C. J. Martyniuk, "Paraquat affects mitochondrial bioenergetics, dopamine system expression, and locomotor activity in zebrafish (Danio rerio)," Chemosphere, vol. 191, pp. 106-117, 2018.

[80] K. Wang, S. Chen, C. Zhang et al., "Enhanced ROS production leads to excessive fat accumulation through DAF-16 in _Caenorhabditis elegans_, Experimental Gerontology, vol. 112, pp. 20-29, 2018.

[81] C. E. Schaar, D. J. Dues, K. K. Spielbauer et al., "Mitochondrial and cytoplasmic ROS have opposing effects on lifespan," PLoS Genetics, vol. 11, no. 2, p. e1004972, 2015.

[82] S. Lei, L. Zavala-Flores, A. Garcia-Garcia et al., "Alterations in energy/redox metabolism induced by mitochondrial and environmental toxins: a specific role for glucose-6phosphate-dehydrogenase and the pentose phosphate pathway in paraquat toxicity," ACS Chemical Biology, vol. 9, no. 9, pp. 2032-2048, 2014.

[83] C. T. Murphy and P. J. Hu, "Insulin/insulin-like growth factor signaling in C. elegans," WormBook: The Online Review of C. elegans Biology, WormBook, 2005.

[84] J. R. Friedman and J. Nunnari, "Mitochondrial form and function,” Nature, vol. 505, no. 7483, pp. 335-343, 2014. 


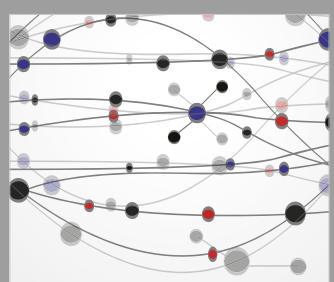

The Scientific World Journal
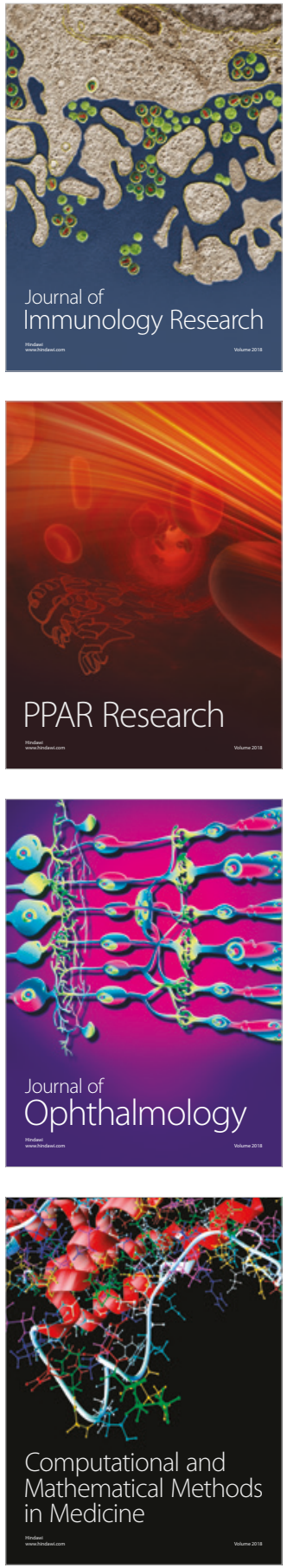

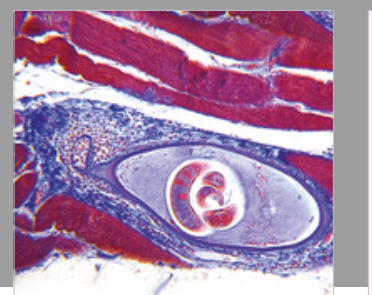

Gastroenterology Research and Practice

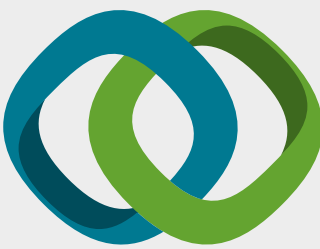

\section{Hindawi}

Submit your manuscripts at

www.hindawi.com
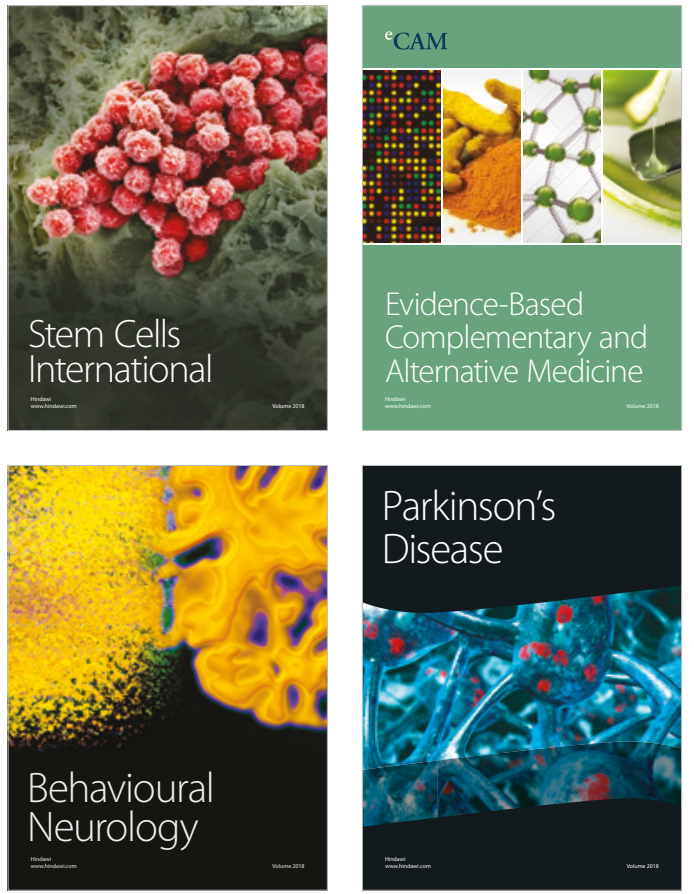

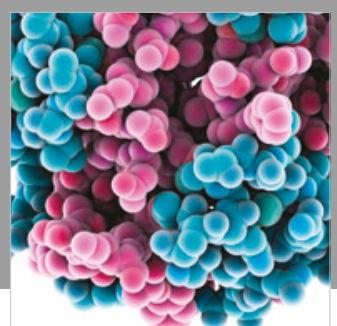

ournal of

Diabetes Research

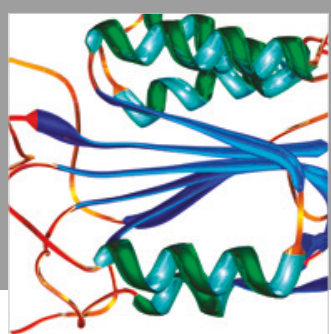

Disease Markers
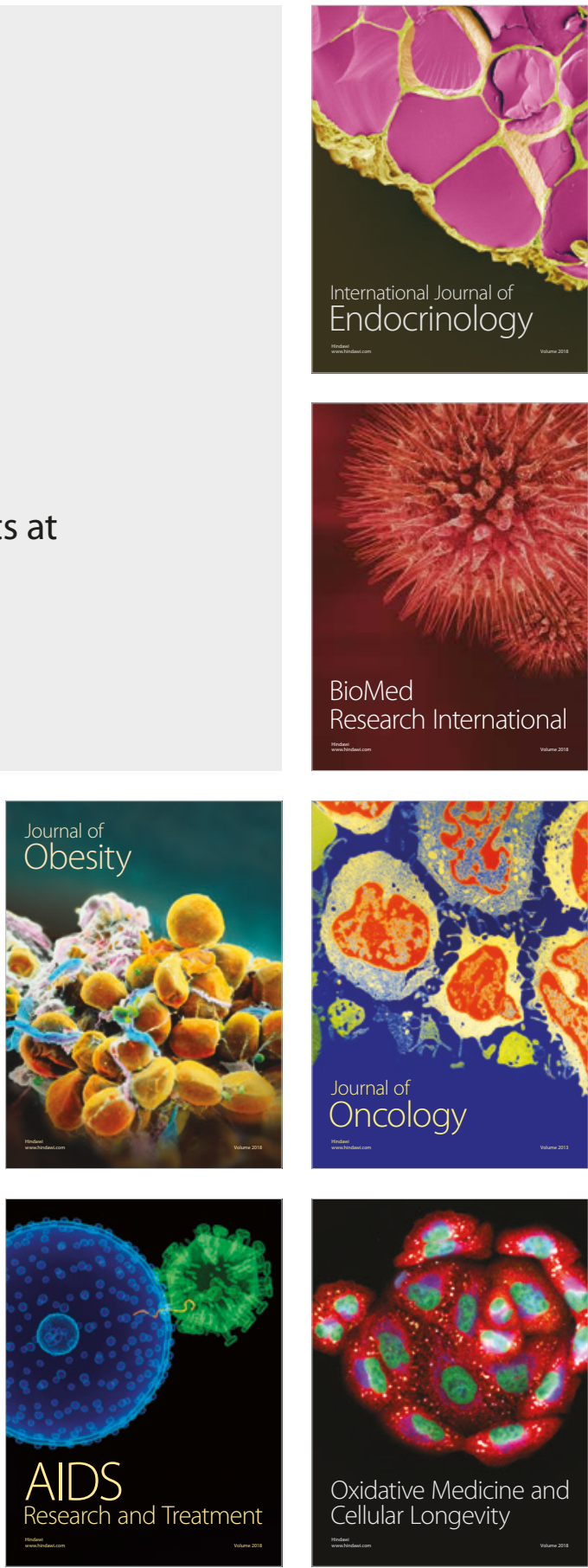